\title{
Protein-based, but not viral vector alone, HIV vaccine boosting drives an IgG1-biased polyfunctional humoral immune response
}

\author{
Stephanie Fischinger, ${ }^{1,2}$ Sally Shin, ${ }^{1}$ Carolyn M. Boudreau, ${ }^{1,3}$ Margaret Ackerman, ${ }^{4}$ \\ Supachai Rerks-Ngarm, ${ }^{5}$ Punnee Pitisuttithum, ${ }^{6}$ Sorachai Nitayaphan, ${ }^{7}$ Jerome H. Kim, ${ }^{8}$ \\ Merlin L. Robb, ${ }^{9,10}$ Nelson L. Michael, ${ }^{10}$ Robert J. O'Connell, ${ }^{7,10}$ Sandhya Vasan, ${ }^{7,9,10}$ \\ Hendrik Streeck, ${ }^{2,11}$ and Galit Alter ${ }^{1}$ \\ 'Ragon Institute of MCH, Harvard and MCH, Cambridge, Massachusetts, USA. ${ }^{2}$ Institut für HIV Forschung, Universität \\ Duisburg-Essen, Essen, Germany. ${ }^{3} \mathrm{PhD}$ Program in Virology, Harvard University, Boston, Massachusetts, USA. ${ }^{4}$ Thayer \\ School of Engineering, Dartmouth College, Hanover, New Hampshire, USA. ${ }^{5}$ Department of Disease Control, Ministry of \\ Public Health, Nonthaburi, Thailand. ${ }^{6}$ Vaccine Trial Centre, Faculty of Tropical Medicine, Mahidol University, Bangkok, \\ Thailand. ${ }^{7}$ Armed Forces Research Institute of Medical Sciences, Bangkok, Thailand. ${ }^{8}$ International Vaccine Institute, \\ Seoul, South Korea. ${ }^{9}$ Henry M. Jackson Foundation for the Advancement of Military Medicine, Bethesda, Maryland, USA. \\ ${ }^{10}$ US Military HIV Research Program, Walter Reed Army Institute of Research, Silver Spring, Maryland, USA. "Institute of \\ Virology, Universitätsklinikum Bonn, Bonn, Germany.
}

\begin{abstract}
The RV144 HIV-1 vaccine trial results showed moderate reduction in viral infections among vaccinees as well as induction of antibody-dependent cellular cytotoxicity and vaccine-specific IgC and IgC3 responses directed at variable loop regions 1 and 2 of the HIV envelope protein. However, with the recent failure of the HVTN 702 clinical trial, comprehensive profiling of humoral immune responses may provide insight for these disappointing results. One of the changes included in the HVTN 702 study was the addition of a late boost, aimed at augmenting peak immunity and durability. The companion vaccine trial RV305 was designed to permit the evaluation of the immunologic impact of late boosting with either the boosting protein antigen alone, the canarypox viral vector ALVAC alone, or a combination of both. Although previous data showed elevated levels of IgC antibodies in both boosting arms, regardless of ALVAC-HIV vector incorporation, the effect on shaping antibody effector function remains unclear. Thus, here we analyzed the antibody and functional profile induced by RV305 boosting regimens and found that although IgC1 levels increased in both arms that included protein boosting, IgC3 levels were reduced compared with the original RV144 vaccine strategy. Most functional responses increased upon protein boosting, regardless of the viral vector-priming agent incorporation. These data suggest that the addition of a late protein boost alone is sufficient to increase functionally potent vaccine-specific antibodies previously associated with reduced risk of infection with HIV.
\end{abstract}

Conflict of interest: The authors have declared that no conflict of interest exists.

Copyright: ( 2020 , American Society for Clinical Investigation.

Submitted: November 19, 2019

Accepted: May 7, 2020

Published: June 18, 2020

Reference information: /CI Insight. 2020;5(12):e135057.

https://doi.org/10.1172/jci.

insight.135057.

\section{Introduction}

Treatment options for HIV-1 and AIDS have reversed the death sentence once associated with an HIV diagnosis. However, global control and eradication of the disease are hindered by limited access to medication, adherence to daily medication, and health care for some populations (1). Additionally, HIV-infected people on long-term treatment continue to experience a number of health complications, including increased risk for particular cancers, cardiovascular disease, and liver damage (2). Therefore, the development of a vaccine that demonstrates protection against HIV remains a public health priority for eradicating the pandemic $(3,4)$.

To date, 4 HIV vaccine phase II and III trials have been conducted, with only one showing a modest level of reduction with infection acquisition. RV144, also known as the Thai trial, was a phase III community-based HIV-1 vaccine trial that showed $60.5 \%$ and $31.2 \%$ efficacy 12 and 42 months after final vaccination (NCT00223080; refs. 5, 6). Despite the absence of neutralizing antibodies, analyses of immune correlates of reduced risk of infection pointed to an enrichment of high levels of IgG1 and IgG3 
responses to variable loop regions 1 and 2 (V1V2) of the HIV envelope (Env) protein and to lower levels of vaccine-induced IgA among uninfected compared with infected vaccinees (7). Moreover, additional analyses revealed enhanced complement deposition in RV144 vaccinees associated with reduced risk of infection (8), as well as more polyfunctional vaccine responses compared with previous protein-based vaccine trials (9). Collectively, these data pointed to the critical importance of functional antibodies, correlated with an array of Fc-mediated effector functions in reduced HIV acquisition risk, following the RV144 prime and boost vaccine regimen.

Owing to the promising but short-lived efficacy of RV144, it was hypothesized that the addition of late boosts might augment the vaccine response and drive a more durable functional immune response. Thus, boosts were added to the HVTN 702 efficacy trial recently terminated in South Africa (10). The disappointing termination of the HVTN 702 trial, owing to futility, raises the possibility that the original RV144 correlates from a low-risk population may not have been sufficiently robust against higher-risk exposure in young women in Africa, or that alterations in the regimen, owing to added boosts, may have altered the humoral immune response, providing less protection from infection in the field.

To determine differences in RV144 and newly boosted vaccine-induced immune responses, we focused on the RV305 trial, developed to investigate whether late boosts with AIDSVAX protein alone, ALVACHIV vector vaccine alone, or a combination of both could selectively augment antibody correlates of reduced risk of infection found in $\operatorname{RV} 144(11,12)$, with the hypothesis that the innate stimulatory vector functions could potentially have critical value for driving enhanced functional antibodies. The RV305 trial reenrolled 162 RV144 vaccinees, who completed the whole vaccination schedule 6 to 8 years after the follow-up period of RV144, in a placebo-controlled, double-blinded trial. Subjects were randomized into 1 of 3 groups that received 2 boosts with either canarypox viral vector (ALVAC) alone, the AIDSVAX B/E gp120 Env protein alone, or a combination of both (13). RV305 analyses pointed to the critical role of the AIDSVAX B/E protein, but not to the ALVAC viral vector in inducing humoral responses. The boost drove higher antibody titers against various Env antigens (gp120 and V1V2), above levels observed at the peak RV144 vaccine time point in plasma as well as in mucosal secretions (14). Moreover, antibody titers were shown to increase after the first but not after the second boost in RV305 vaccinees. Unfortunately, late boosting did not induce a more durable antibody response, as responses waned quickly after boosting across all groups (13). Furthermore, analysis of B cell responses in RV305 vaccinees showed that a pool of Env CD4-binding site reactive memory B cells were expanded and produced antibodies with longer third heavy chain complementarity-determining regions (14), pointing to a potentially advantageous level of affinity maturation and B cell receptor evolution. However, whether the different boosting strategies altered the qualitative profile of the vaccine-elicited antibody response and antibody functionality of the originally primed RV144-induced vaccine response remains unknown. Thus, here we used a systems serology approach to comprehensively dissect the qualitative profiles induced across the RV305 vaccine groups to gain a deeper sense of protein, viral vector, or combined vaccine boosting impact. We demonstrate that boosting with ALVAC-HIV alone did not result in increased antibody levels or vaccine-induced functional humoral immune responses compared with RV305 placebo recipients. In contrast, delayed protein boosting drove robust functional humoral immune responses, shifting away from IgG3, through the induction of functionally enhanced $\operatorname{IgG} 1$ responses.

\section{Results}

The goal of the RV305 trial was to assess the impact of booster immunizations 6-8 years after completion of RV144 on immune responses (Figure 1A). RV144 vaccinees were reboosted with 2 additional administrations of either ALVAC-HIV or AIDSVAX B/E alone or a combination of both.

Antibody subclass responses are similar across boosting approaches. Previous RV305 studies highlighted the presence of higher levels of IgG1 and IgA after boosting compared with the RV144 peak immunogenicity time point. Moreover, both neutralizing antibodies against tier 1 viruses and increased $\mathrm{CD} 4^{+}$functionality were observed (13). However, comparative studies of RV144 and the failed AIDSVAX B/E vaccine trial VAX003 suggested that changes in antibody subclass levels, associated with the selection of more functional IgG1 and IgG3 antibody subclasses (9), were associated with reduced risk of infection among RV144 vaccinees (12). Thus, we initially sought to define whether the addition of the viral vector priming ALVACHIV immunogen, owing to its potentially adjuvanting viral properties, could enhance and/or shift subclass selection during the boosting phase. Therefore, subclass V1V2 and gp120 levels were assessed against the 
A

\section{RV144}

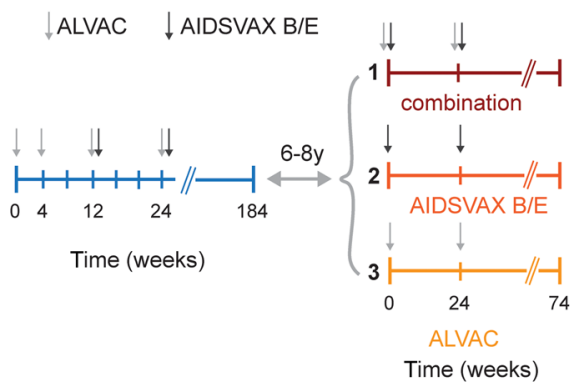

B

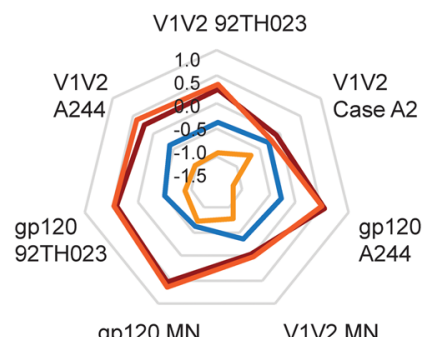

$\lg$ G3

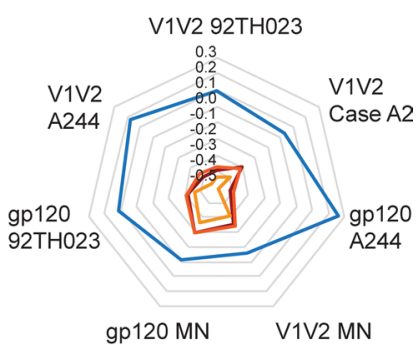

C
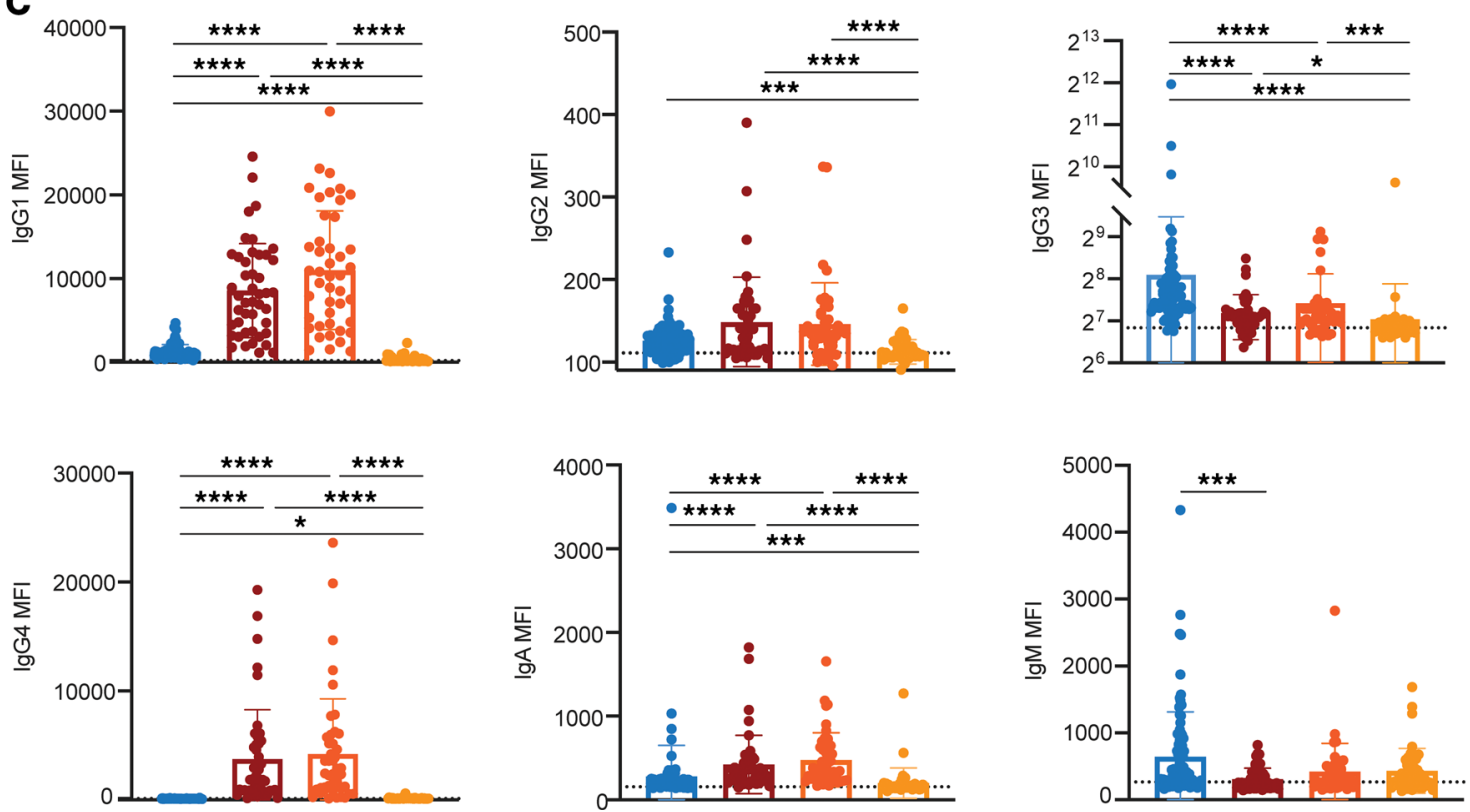

Figure 1. AIDSVAX protein boosting alone or in combination with ALVAC drives an IgG1-focused immune profile. (A) The diagram shows the vaccination schedule of RV144 linked to RV305. RV305 reenrolled healthy RV144 participants and administered 2 boosts of AIDSVAX B/E, ALVAC, or a combination of the two. (B) Spider plots show the relative antibody titers MFIs (z-scored) via Luminex against different vaccine antigens at week 26 for RV144 (blue), combination (maroon), AIDSVAX (orange) and ALVAC (yellow) for IgG1 (left) and IgG3 (right). Each line represents the median of the z-scored values for each vaccine group of the isotype MFI. (C) The dot plots show relative gp120MN-specific antibody level MFIs across the subclasses and isotypes (IgG1, IgG2, $\operatorname{lgG3}$, IgG4, IgA, and IgM). Each dot represents one vaccinee. Data are shown as mean $\pm \mathrm{SEM}$. A Kruskal Wallis test was performed to test for statistical differences across groups. ${ }^{*} P<0.05,{ }^{* *} P<0.01,{ }^{* *} P<0.001$, and ${ }^{* * *} P<0.0001$. The dotted line shows placebo levels.

canarypox-expressed gp120 (92TH023) and AIDSVAX B/E gp120 (MN and AE244) antigens. As previously shown, IgG1 responses were robustly boosted but exhibited similar antigenic profiles in the AIDSVAX $\mathrm{B} / \mathrm{E}$ and combination arms (Figure 1B). However, marginal increases in IgG3 were observed upon boosting across either arm (Supplemental Figure 1; supplemental material available online with this article; https:// doi.org/10.1172/jci.insight.135057DS1), in contrast to RV144 vaccinees who possessed significantly higher IgG3 levels at peak immunogenicity (Figure 1B). Moreover, IgG1 and IgG3 levels were not boosted in the arm using ALVAC-HIV alone (Figure 1B). Slight, insignificant differences were observed in V1V2 responses in the AIDSVAX B/E and combination groups, with slightly elevated V1V2 A244 and lower V1V2 Case A2 responses in the AIDSVAX B/E alone group (Figure 1B).

Addition of the protein antigen is key to enhancing gp $120 \mathrm{IgG}$ levels. Because differences in subclass selection profiles were associated with reduced risk of infection in the RV144 trial, a full analysis of antibody profiles was conducted across the RV305 arms. Across all antibody subclasses and isotypes, individuals immunized with ALVAC-HIV alone had low antibody levels comparable to placebo-immunized individuals, suggesting that reboosting with the ALVAC vector vaccine did not elicit a detectable antibody response, consistent with previous reports (ref. 13; Figure 1C). AIDSVAX alone or in combination with ALVAC induced 
equal levels of IgG1, IgG2, IgG4, and IgA at higher levels than that observed in the original RV144 peak response. These observations indicate a beneficial effect of repeated boosting with a protein antigen to increase antibody titers. IgM levels were comparably low across all vaccine groups, despite higher levels in some RV144 vaccinees compared with the combination boost arm. This points to a maturation of the naive HIV-specific response among all AIDSVAX-boosted individuals, although comparably higher overall titers among individuals receiving the protein antigen were observed.

Notably, in previous analyses of immune correlates, HIV-specific IgG3 levels were associated with reduced risk of infection (12), and IgA was associated with enhanced risk of infection (11). All boosting strategies failed to increase IgG3 responses (Figure 1C), highlighting a maturation and selection of potential memory HIV-specific IgG responses, since IgG1 and IgG4 levels were boosted. Boosting with protein, but not viral vector ALVAC-HIV alone, resulted in increased IgA levels (Figure 1C) along with higher IgG4, both thought to interfere with IgG1 and IgG3 antibody functional potency (9). Thus, collectively, boosting significantly skews the responses away from more naive IgG isotypes and/or subclasses (IgM and IgG3). However, protein boosting was required to drive enhanced functional antibody selection.

Combination and AIDSVAX induce highest functional levels. Given these differences in antibody subclass selection, we next determined whether the functional profiles of the vaccine-induced immune response shifted with titers or were qualitatively enhanced in the combination boost arm. For most functional measurements, combination and AIDSVAX alone induced the highest levels across all functional assays (Figure 2A). Both protein-containing boosting arms and RV144 vaccinees exhibited enhanced vaccinespecific function across all assays (Figure 2A), suggesting that ALVAC-HIV alone did not enhance antibody effector function in any appreciable manner across all functional measurements. Yet, protein boosting only significantly increased neutrophil phagocytosis (ADNP), complement deposition (ADCD), and NK cell chemokine secretion compared with RV144 vaccination alone (Figure 2A). These data suggest that late boosting returns antibody-dependent monocyte phagocytosis (ADCP), NK cell degranulation (CD107a), and IFN- $\gamma$ back to RV144 levels, while significantly boosting other innate immune effector functions. Although previous studies have shown that CD107a expression correlated with cytokine secretion (15), different NK cell subsets show differences in their capacity to produce cytokines (16). Therefore, different cell subsets could be activated differentially by vaccination, resulting in CD107a expression, IFN- $\gamma$, and MIP-1 $\beta$ secretion differences. MIP-1 $\beta$ secretion typically induced at highest rates after stimulation and was most sensitive to differences in antibody quality (17). Interestingly, correlational analysis between vaccine-induced isotype and/or subclasses and effector functions (Figure 2B) pointed to the dominant role of IgG1 in driving antibody effector functions across RV144 and protein-containing boosting arms. However, IgG3 levels in RV144 vaccinees were significantly correlated with ADCD, and only weakly, nonsignificantly correlated with ADCP, ADNP, NK degranulation, and NK IFN- $\gamma$ secretion. These relationships were nearly eliminated with boosting in the combination and AIDSVAX-only arms. Instead, weak relationships were observed with other isotypes and/or subclasses, including IgA heterogeneous associations with ADCP, ADNP, ADCD, and NK cell functions across the 2 protein boosting arms that did not reach significance after Bonferroni's correction. Given the constitutive expression of the F $\mathrm{c} \alpha \mathrm{R}$ on neutrophils, the direct functional role of IgA can only be examined in this readout; however, the influence of $\operatorname{IgA}$ on functions in collaboration with $\operatorname{Ig} \mathrm{A}$ is possible.

These data support the argument for a consistently dominant role of IgG1 in driving antibody function across all vaccine groups that may be fine-tuned in the setting of additional antibody isotypes and/or subclasses. Thus, whereas boosting appears to improve the overall functional profile of the humoral immune response, limited differences were observed again at the univariate level across the protein-containing boosting groups.

We next investigated whether the vaccine regimens altered the durability of the functional response. Therefore, functional profiling was performed at baseline, peak immunogenicity (week 24), and at a later time point (week 72). Although the functions did not increase significantly over the follow-up among ALVAC-only vaccinees, ADCP, ADNP, and ADCD clearly peaked at peak immunogenicity (Figure 2C). Protein-boosted groups showed similar trends across all functions, with ADNP and ADCD rising and falling to baseline levels by week 72. Conversely, ADCP activity remained elevated above background at week 72, suggesting that some, but not other, functions may persist for longer periods of time in both protein-boosted groups. These data point to indistinguishable trajectories of the vaccine-induced functional humoral immune response over time with a persistent and durable ADCP response. 
A
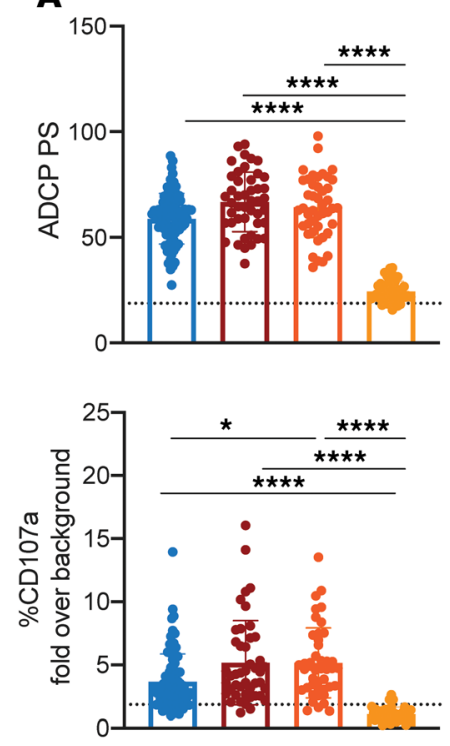
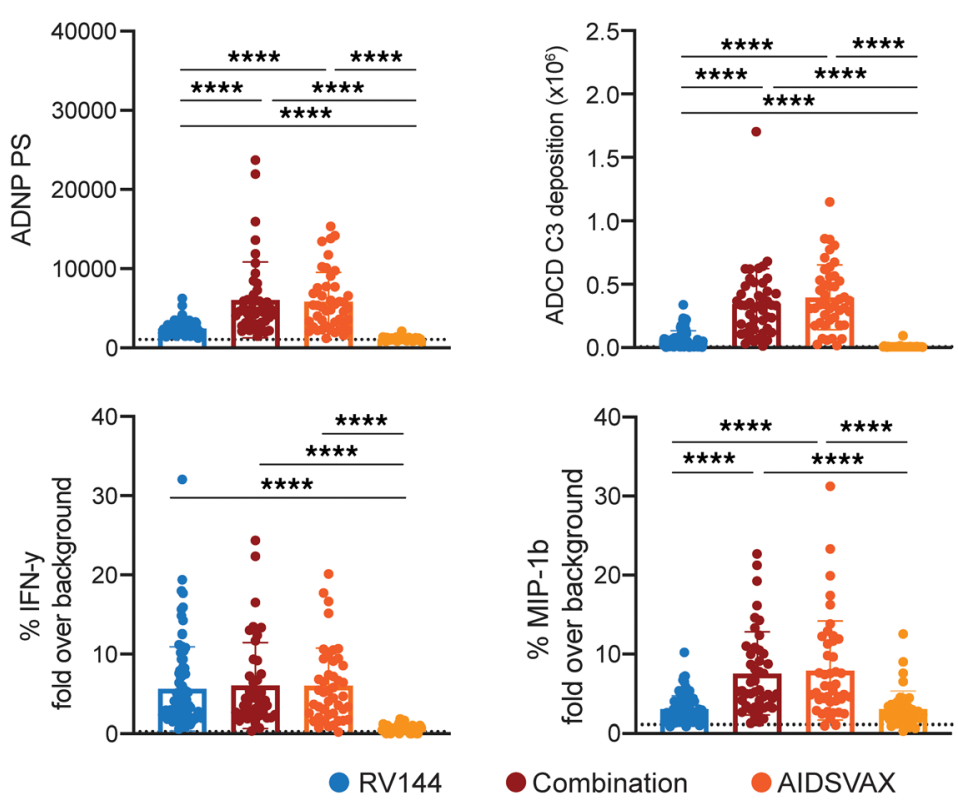

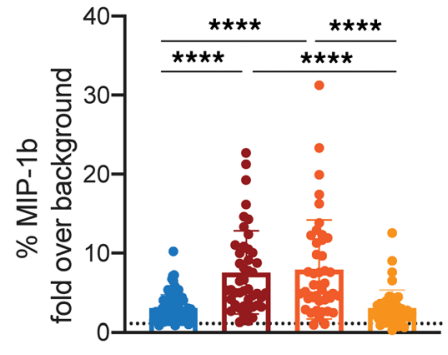

- Combination AIDSVAX ALVAC
B
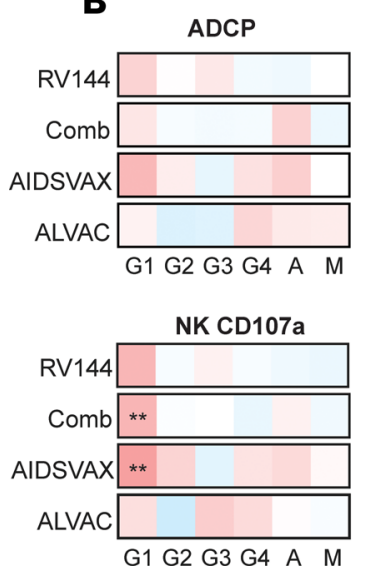

ADNP

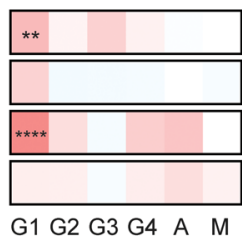

NK IFN-y

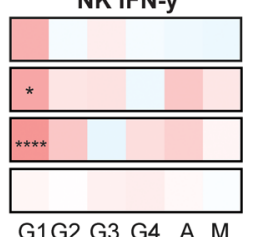

ADCD

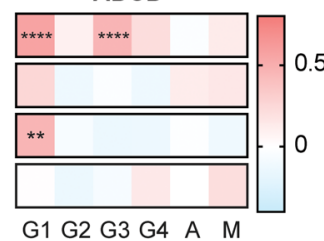

NK MIP-1b

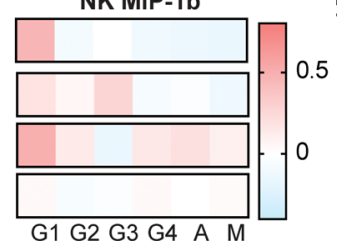

C
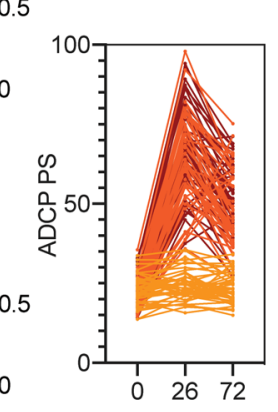
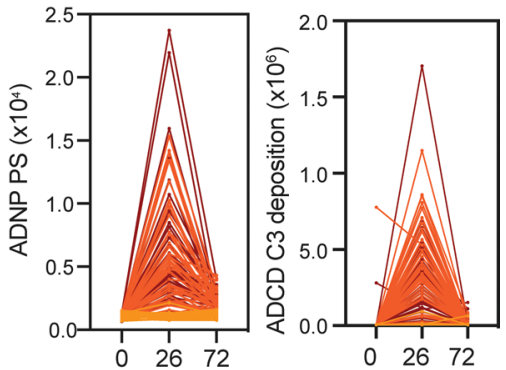

Figure 2. Late boosting with AIDSVAX or ALVAC/AIDSVAX elevated ADNP, ADCD, and MIP-1ק and resulted in more durable ADCP. (A) The dot plots show functional responses for the different vaccine groups against gp120 MN. Each dot represents one vaccinee. Data are shown as mean \pm SEM. ADCP, PS, ADNP PS, ADCD, MFI, and ADNKA (percent positive NK cells for CD107a, IFN- $\gamma$, and MIP-1 $\beta$ ) are depicted. Dotted lines indicate the average activity in the placebo vaccinees. (B) Heatmaps show Spearman's correlations for each functional measurement with Luminex antibody levels for: RV144, combination, AIDSVAX, and ALVAC. The color indicates the Spearman r value: positive correlation (red), negative correlation with a Bonferroni's adjustment for multiple comparisons (blue), ${ }^{*}<0.05$, ${ }^{* *} P$ $<0.01,{ }^{* *} P<0.001$, and ${ }^{* * *} P<0.0001$. (C) The dot plots show ADCP, ADNP, and ADCD over time, starting at week 0 (baseline), to week 26 ( 2 weeks post second boost) week 72 (durability time point). Each graph shows group 1 (dark red), 2 (orange), and 3 (yellow) for each function, and the same individuals are connected by lines. ADCD, antibody-dependent complement deposition; ADCP, Antibody-dependent cellular phagocytosis; ADNKA, antibody-dependent NK cell activation; ADNP, antibody-dependent neutrophil phagocytosis; PS, phagoscore.

Combination and AIDSVAX induce significantly different immune profiles from RV144 and ALVAC. Despite the lack of univariate differences across the boosting arms, we next aimed to determine whether multivariate differences could be discerned across the groups. A supervised multivariate analysis was applied to the peak RV305 and RV144 data. Canonical discriminant analysis, unlike principal component analysis, is a supervised multivariate analysis that explores the linear combinations of quantitative variables that provide maximal separation between groups. Using this discriminant analysis, with all 118 data points, including antigen-specific functional, isotype, subclass, and Fc receptor (FcR) measurements for each subject $(n=247)$, clear separation was observed across several groups (Figure 3A). Interestingly, RV144 split as a completely independent profile from all other vaccine groups; placebo and ALVAC-only boosted individuals were inseparable in this analysis. Conversely, the 2 arms that received the protein AIDSVAX boost clustered together as overlapping profiles. These data clearly illustrate the marginal effect of the ALVAC vector boost alone, but the critical impact of the protein boost in reshaping the RV144 vaccine induced immune response. 
Despite the limited separation between the AIDSVAX only and ALVAC/AIDSVAX arms by univariate and discriminant analysis, we next aimed to use a more rigorous multivariate approach to determine whether any differences existed between the 2 vaccine arms. Using a multivariate LASSO partial least squares discriminant analysis (PLSDA) model, in which all antibody features were first down selected to prevent overfitting, and to define the minimal features that distinguish the arms, some level of separation was observed between the combination and AIDSVAX arms (Figure 3B). Both regimens resulted in distinct V1V2 immunodominant reactivity profiles that had been previously associated with reduced risk of infection (11). Although the combination vaccine drove higher levels of IgG2 breadth of binding, IgM responses to V1V2, IgG4 responses, and NK cell responses, AIDSVAX drove an FcR -biased response to Clade $\mathrm{AE}$ antigens, higher complement $\mathrm{C} 1 \mathrm{q}$ binding, as well as IgG1 titers to the Case A2 V1V2 (Figure 3C). Whereas many of these were not significantly different at a univariate level, the combination of a number of features were ultimately able to separate out the vaccine profiles, highlighting subtle, largely epitope-specific functional differences across the vaccine responses. Therefore, these higher-resolution analyses point to fine-detailed differences in the immune response; however, how these differences are relevant clinically remains unclear.

Finally, to gain a deeper understanding on how the boosting reshaped the RV144 vaccine-induced immune response, RV144 and the matched combination arm were compared in a LASSO PLSDA model. RV144 and RV305 AIDSVAX/ALVAC immunized individuals exhibited completely distinct, nonoverlapping functional humoral immune profiles (Figure 4A) marked by elevated binding to FcR 2A (Figure 4 , $\mathrm{B}$ and $\mathrm{C}$ ) involved in phagocytosis (18), complement binding, and less desirable elevated IgG4 responses. Conversely, RV144 vaccinees possessed higher IgG3 breadth and IgM responses, both of which were lost with additional boosting as observed in the univariate analysis (Figure 4, B and C). Thus, whereas the potentially protective IgG3 response was lost from the RV144 profile in RV144 vaccinees, the boost matured the immune response to a robust FcR--binding profile poised to drive antibody effector function. The small number of 8 features needed for separation between the RV144 and RV305 profiles point toward qualitative rather than primarily quantitative differences in the immune response tailored by boosting. After late boosting in RV305, regardless of the protein immunization strategy, these individuals were able to leverage more FcR 2A binding, skewing the humoral response toward a highly functional but low IgG3 profile.

\section{Discussion}

Reduced rates of infection among RV144 vaccinees provided great hope to the HIV vaccine research field that protection could potentially be achieved (5). However, the RV144 replication in South Africa, the HVTN 702 trial, was recently terminated due to futility (10). Given the differences established in the vaccine regimens across the studies (19), with the addition of the late boost in the HVTN 702 study, here we sought to comprehensively dissect the effect of late boosting on vaccine-induced immunity. Owing to issues with waning immunity following RV144 vaccination (5), the RV305 study aimed to test the immunological impact of boosting that was included in the HVTN 702 trial. Within the RV305 study, the potential adjuvanting activity of ALVAC-HIV as well as the protein boost were explored. Whereas previous studies showed that IgG1 and IgA levels increased compared with RV144 peak immunogenicity levels in AIDSVAX and combination vaccine groups, to date functional responses as well as subclass distributions had not been thoroughly dissected (13). To further evaluate RV305-induced vaccine profiles, using systems serology we confirmed that protein boosting alone significantly boosted vaccine responses. At a univariate level, protein boosting alone, regardless of ALVAC addition, resulted in significant boosting at both quantitative and qualitative levels, in an IgG-dominated manner. Although boosting did not augment the overall level of ADCP and NK cell degranulation or IFN- $\gamma$ secretion by vaccine-induced antibodies, boosting augmented ADNP, ADCD, and NK cell-mediated chemokine secretion, pointing to qualitative improvements with the addition of the boosting. Moreover, whereas the majority of antibody functions declined quickly upon follow-up, ADCP responses remained significantly elevated, indicating additional durability in some functional vaccine-induced immunity with boosting.

Whereas IgG3, the most functional antibody subclass in humans, was identified as an immune correlate of reduced risk of infection in the RV144 vaccine trial (12), all boosting strategies in RV305 drove class switching away from this functional subclass (Figure 1C). The boosted IgG response was largely more effective at driving functional activity compared with the RV144 responses, suggesting that despite this loss of IgG3, the increased IgG1 titers were highly functional (Figure 2A), albeit less functional at a per 
A

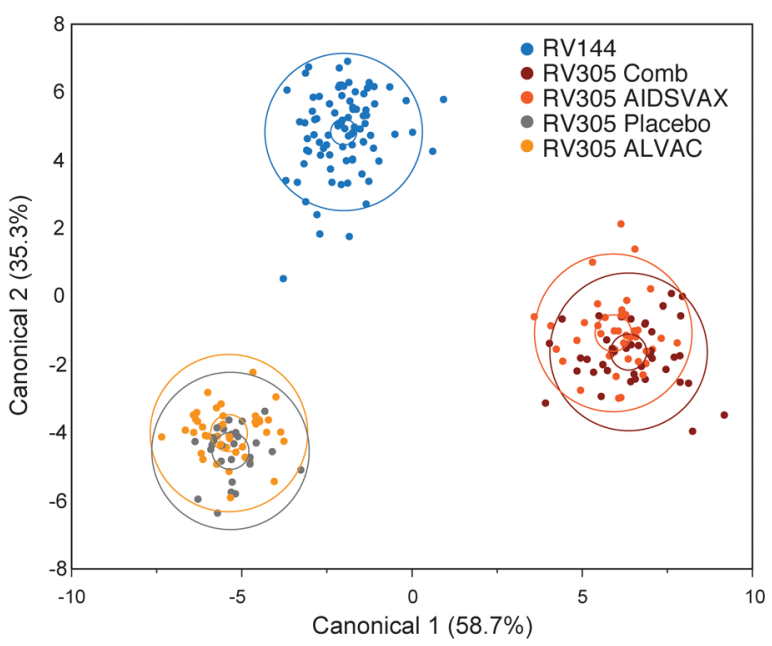

B

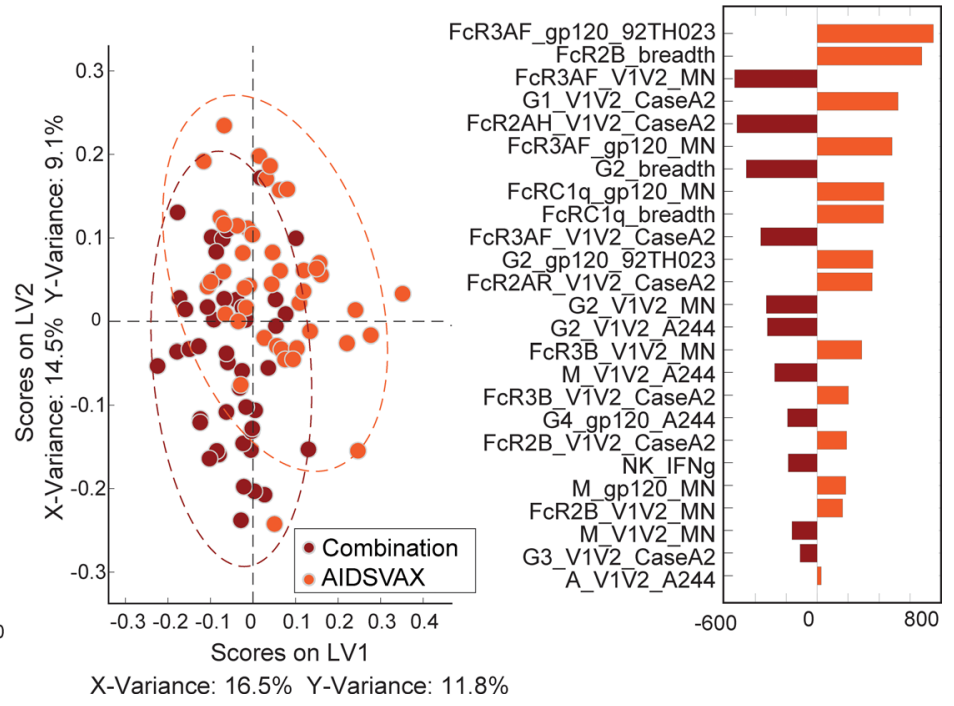

Figure 3. Multivariate determinants of differences across boosting regimens. A total of 118 data points, including functional measurements for gp120 MN and all antigens described in "Methods" for Fc-receptor and isotyping and/or subclassing, have been used for multivariate analysis. (A) The discriminant analysis shows the separation of RV144 and RV305 vaccine arms, including the RV305 placebo subjects. (B) The LASSO partial least squares discriminant analysis shows the separation between the RV305 combination vs. AIDSVAX alone vaccine recipients. Each dot represents one participant. (C) The variable importance in the projection scores are ordered and scaled according to their importance for model separation. Variables that point to the right are enriched in AIDSVAX-only recipients, whereas the features directed to the left are higher in recipients of the combination vaccine.

antibody level compared with those elicited in the RV144 vaccine trial. Likewise, depletion IgG3 studies led to a loss of function in RV144 vaccinees, highlighting the importance of IgG3 in polyclonal RV144 functionality (9). However, IgG3 depletion did not completely deplete polyclonal functionality, pointing to a critical role for IgG1 as a critical mediator of vaccine-induced effector function. Given the short IgG3 half-life (20), the induction of more functional IgG1 is likely to be a more stable target vaccine profile for future vaccines; however, with IgG3, polyclonal pools may have the capacity to drive enhanced antibody effector function. Thus, vaccine strategies that eliminate IgG3 may fail to fully leverage all necessary antibody effector functions, such as ADCD that was tightly linked to IgG3 levels, which may be required for long-lasting protection against HIV.

In addition to boosted IgG1 levels, boosting with either combination or AIDSVAX alone also increased IgG4 levels, which have been associated negatively with antibody functionality (21). Previous studies have shown that repeated boosting, as was performed in VAX003, results in class switching and increase of less-functional IgG4 antibodies (9). However, adjuvant use may temper the level of subclass selection, potentially offering an approach to enhance the functional quality of the vaccine-induced humoral immune response. For example, whereas VAX003 and RV144 used alum as an adjuvant, the use of MF59 and AS01 as adjuvants has demonstrated induction of superior antibody functionality and increased IgG1 levels (22). In a previous study, using a MF59-adjuvanted influenza vaccine demonstrated the induction of robust IgG1, along with increasing IgG4 titers (23). Surprisingly, although MF59 induced robust phagocytic and complement fixing antibodies in parallel to increasing antibody titers, the vaccine-induced immune response lacked the ability to recruit NK cells, a function that has been linked to reduced risk of infection in the RV144 correlates analyses (11). Therefore, the lack of efficacy observed in HVTN 702 may be related to altered antibody functional profiles driven both by changes in boosting as well as in adjuvant selection.

Although the majority of functions declined after peak immunogenicity, ADCP remained significantly elevated (Figure 2C). Emerging data from multiple nonhuman primate vaccine studies, using distinct immunization strategies, argue for a critical protective role of phagocytosis against infection $(24,25)$. Although ADCP levels did not increase significantly in RV305 compared with RV144 vaccinees, the durability of ADCP in the protein-boosted subjects may offer extended protection. How these ADCP responses persisted in the setting of rapidly waning IgG titers (13) remains to be defined, but points to the differences in quantitative and qualitative vaccine-induced immune persistence that may greatly impact the durability of protection. Thus, whereas late boosting failed to introduce long-lived IgG responses (13), late boosting 
A

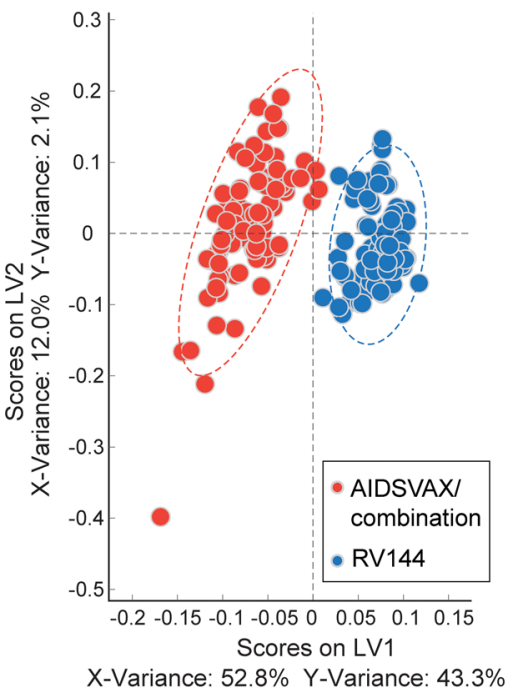

B

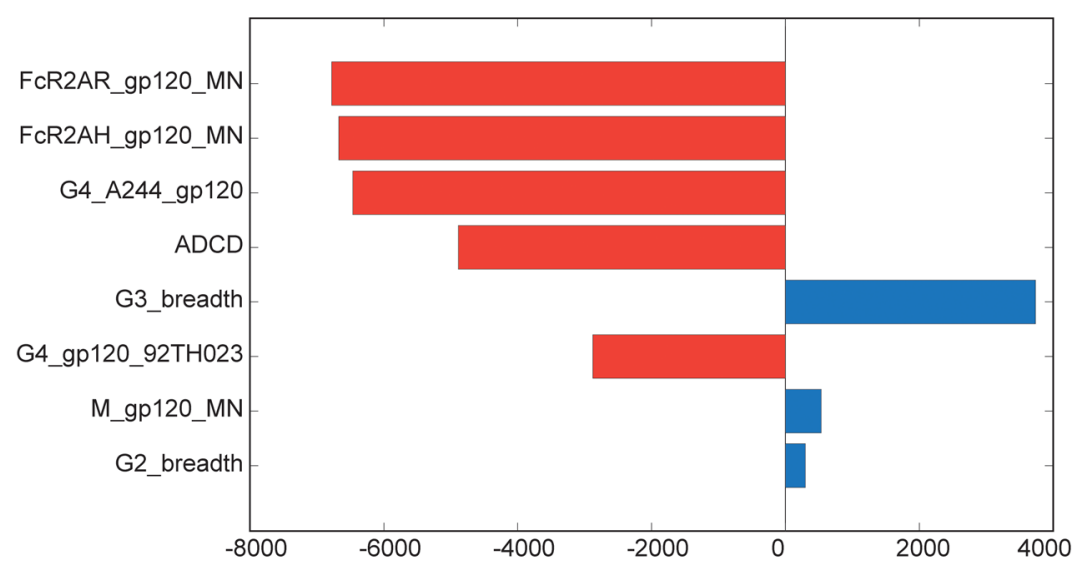

C

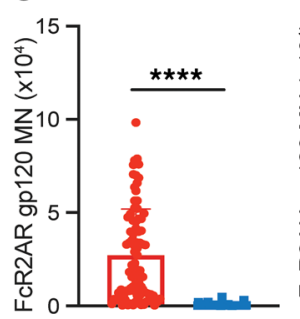

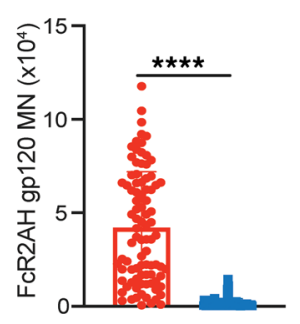
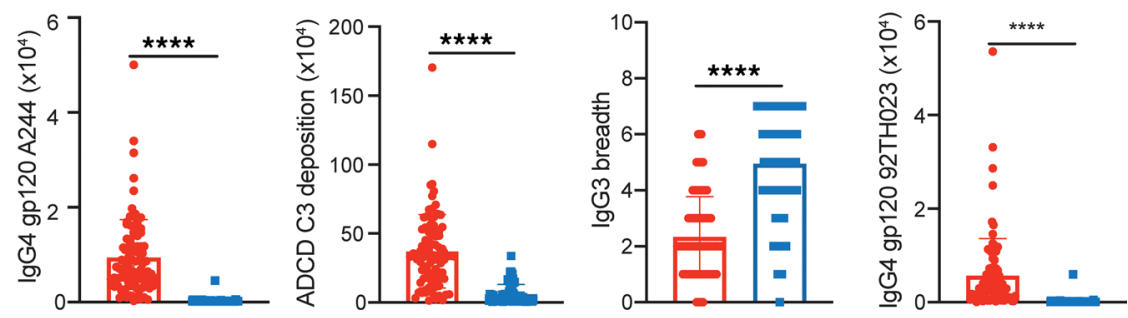

Figure 4. RV144 drove an IgG3-focused but less functional profile compared with vaccinees boosted with AIDSVAX boost alone or in combination with ALVAC-HIV. (A) The partial lease squares discriminant analysis plot depicts the features associated with separation of RV305 (combination and AIDSVAX recipients) and RV144 vaccinees. Each dot represents one participant with all measurements, including measures from all antigens and Fc receptor binding. (B) The variable importance in the projection scores are ranked according to their importance, and direction corresponds to enrichment in each group. (C) The dot plots show the top 6 variable importance in the projection features as univariate comparison between the AIDSVAX/combination and RV144 group. Mann-Whitney test was performed with a Bonferroni's correction to define significance. ${ }^{* * *} P<0.0001$.

with protein antigens resulted in a profound improvement in antibody effector function and the persistence of elevated levels of ADCP (Figure 2, A and C); however whether these persisting responses may not have been sufficiently high to confer protection in HVTN 702 remains to be defined. Collectively, subtle differences existed in the vaccine-induced immune profile across the protein alone and combination of viral vector ALVAC and protein boost, but suggested that the protein boost alone may be sufficient to drive highly functional vaccine-induced immune responses (Figure 3, B and C).

Collectively, late boosting altered the overall RV144 vaccine-induced humoral profiles. Given the recent failure of HVTN 702, these data point to the possibility that boost-driven alterations may have skewed the response away from RV144 profiles or that distinct and/or more robust responses may be required to confer protection in the aggressive South African epidemic in young women. However, novel adjuvants (22), poised to drive enhanced functionality, paired with rational late boosting with newly designed structurally and/or sequence-enhanced antigens (26) may result in the generation of an optimized vaccine able to provide higher levels of protection from infection at a global level.

\section{Methods}

Sample selection. Plasma samples from 88 uninfected RV144 vaccinees (5) (NCT00223080) (not matched to RV305 participants) at the peak immunogenicity time point (week 24) were assayed and compared with 132 RV305 vaccinees at week 0, 26, and 72. RV144 vaccinees received 2 priming injections with ALVAC-HIV (encoding vCP1521, Sanofi Pasteur) followed by 2 prime and boost injections with the ALVAC-HIV and AISVAX B/E recombinant protein boost (including gp120 A244 and MN, Global Solutions for Infectious Diseases). The RV305 trial (NCT01435135) reenrolled 162 RV144 vaccinees who completed the whole 
vaccination schedule 6 to 8 years after the follow-up period of RV144 (13). The RV305 study included 3 vaccine arms, each including 45 participants, and 9 individuals in each placebo group. The same priming and boosting vaccines were used as in the RV144 trial: the ALVAC prime and AIDSVAX B/E boost. Group 1 received a combination of the ALVAC-HIV and AIDVAX B/E vaccination (45 individuals available), group 2 received the boosting AIDVAX B/E alone (44 individuals available), and group 3 received ALVAC-HIV alone (43 individuals available). Vaccines were given at week 0 and 24 (Figure 1A). All RV305 samples $(n=132)$ were profiled at week 26 (peak immunogenicity time point) and partially at week $72(n=$ 132). Samples were run in duplicate per assay and across 2 biological replicates.

Antibody titer measurements and $F_{C} R$ binding. Antigen-specific antibody subclass, isotype, and FcR-binding levels were measured using a customized multiplexed Luminex assay, as previously described (27). A panel of V1V2 and gp120 antigens were used, including: V1V2 92TH023, V1V2 A244, V1V2 MN, gp120 92TH023, gp120 MN, and gp120 A244 (all from Duke University) and V1V2 Case A2 (Immune tech, IT-001-214p). Briefly, antigens were coupled to fluorescent carboxyl-modified microspheres (Luminex). Antigen-coupled Luminex beads were then washed and incubated with plasma samples (1:1000 for IgG, IgG1, and all FcRs; 1:250 for all other readouts), and antibody titers were detected using a PE-conjugated antibody for each subclass, isotype (IgG, IgG1, IgG2, IgG3, IgG4, IgA, and IgM; Southern Biotech), FcR (FCR2AH, 2AR, 2B, 3AF, 3AV, and 3B; Duke Protein Production facility), or complement (C1q, Duke Protein Production Facility). Samples were analyzed on a Bioplex 3D reader (Bio-Rad) using Exponent software. Breadth of antibody subclasses and FcR binding was calculated for all mentioned antigens as the number of antigens that bound above the median $\mathrm{z}$-score value for each subclass or FcR, respectively.

Functional measurements. Bead-based assays were used to quantify ADCP, ADNP, and ADCD, as previously described (28-30). Fluorescent streptavidin beads (Thermo Fisher Scientific) were coupled to biotinylated antigen (gp120 MN) and incubated with diluted plasma (ADCP 1:500, ADNP 1:100, and ADCD 1:10). A phagocytosis score was calculated for ADCP and ADNP as (percentage of bead-positive cells) $\times$ (MFI of bead-positive cells) divided by 10,000. ADCD was reported as MFI of C3 deposition.

For antibody-dependent NK cell-activating functions, an ELISA-based surrogate assay was employed, as previously described (31). Briefly, plates were coated with $3 \mu \mathrm{g}$ of antigen (gp120 MN), and samples were added at a $1: 25$ dilution and incubated for 2 hours at $37^{\circ} \mathrm{C}(32)$. NK cells were isolated the day prior via RosetteSep (Stem Cell Technologies) from healthy buffy coats and rested overnight in $1 \mathrm{ng} / \mathrm{mL}$ IL-15 (Stemcell). NK cells were then added to the immune complex containing plates and incubated for 5 hours at $37^{\circ} \mathrm{C}$ in the presence of CD107a PE-Cy5 (BD), Golgi stop (BD), and Brefeldin A (BFA, MilliporeSigma) staining cocktail. Following the incubation, cells were fixed with Perm A (Life Tech), stained for surface markers with anti-CD16 APC-Cy7 (BD), anti-CD56 PE-Cy7 (BD), and anti-CD3 PacBlue (BD), and permeabilized with Perm B (Life Tech). Anti-IFN- $\gamma$ FITC (BD) and anti-MIP-1 $\beta$ PE (BD) antibodies were used for intracellular staining. NK cells were defined as $\mathrm{CD}^{-}, \mathrm{CD}_{16}{ }^{+}$, and $\mathrm{CD}_{5} 6^{+}$. Data were reported as percentage of cells positive for CD107a, MIP-1 $\beta$, or IFN- $\gamma$. Acquisition of all flow data was performed on an iQue Screener plus (IntelliCyt).

Statistics. Flow data was analyzed using the iQue Screener plus (Intellicyte) software. Data analysis and graphs were generated using GraphPad Prism version 8 for Mac (GraphPad Software). For comparison between 2 groups (Figure 4C), 2-tailed Mann-Whitney test was applied. For comparison of more than 2 groups (Figure 1C and Figure 2A), Kruskal-Wallis with Dunn's multiple comparison test was used. Correlation matrixes were generated via Prism, and Spearman's correlation was performed. Bonferroni's post hoc test was applied as indicated for multiple comparisons (Figure 2B and Figure 4C). JMP version 13.2.0 was employed for the linear canonical discriminant analysis with common covariance. The minimum signature of antibody features and functional measurements needed for differentiating vaccine groups were determined using a LASSO feature down-selection using MATLAB software (version 2018B, MathWorks) followed by visualization by PLSDA (33). For RV305 AIDSVAX versus combination (Figure 3), a ROC 10-fold cross-validation was used (72.8\% accuracy, AUC 0.56). For RV144 versus RV305 AIDSVAX/combination analyses (Figure 4), the ROC 1-fold cross-validation yielded $91.5 \%$ accuracy and 0.90 AUC.

Study approval. Written informed consent was received from all participants before inclusion in the study. This research was approved by the Institutional Review Board of Massachusetts General Hospital (IRB approval no. 2015P000095 and no. 2014P000926). 


\section{Author contributions}

SF and GA designed the research study. SF and SS conducted the experiments and acquired the functional data. MA provided additional biophysical data. SF and CMB analyzed the data. SV provided study samples. SF and GA wrote the manuscript. SRN, PP, SN, SV, JHK, MLR, NLM, RJOC, and HS gave valuable comments and input on the manuscript. All authors contributed to the final version of the manuscript.

\section{Acknowledgments}

We would like to thank the US Military HIV Research Program for specimens from the RV144 and RV305 vaccine trial. This work was supported by the NIH under award R37AI080289, the Ragon Institute Sundry, and the Massachusetts General Hospital Executive Committee on Research Scholars Program.

Address correspondence to: Galit Alter, 400 Technology Square, Cambridge, Massachusetts 02139, USA. Phone: 857.268.7003; Email: galter@partners.org.

1. Tafuma TA, et al. Barriers to HIV service utilisation by people living with HIV in two provinces of Zimbabwe: Results from 2016 baseline assessment. South Afr J HIV Med. 2018;19(1):721.

2. Casper C, Crane H, Menon M, Money D. HIV/AIDS Comorbidities: Impact on cancer, noncommunicable diseases, and reproductive health. In: Holmes KK, Bertozzi S, Bloom BR, Jha P, eds. Major Infectious Diseases. Washington, DC, USA The International Bank for Reconstruction and Development/The World Bank; 2017.

3. Prevent HIV, Test and Treat All - WHO Support for Country Impact. World Health Organization Web Site. https://www.who int/hiv/pub/progressreports/2016-progress-report/en/. Accessed May 18, 2020.

4. Day TA, Kublin JG. Lessons learned from HIV vaccine clinical efficacy trials. Curr HIV Res. 2013;11(6):441-449.

5. Rerks-Ngarm S, et al. Vaccination with ALVAC and AIDSVAX to prevent HIV-1 infection in Thailand. N Engl J Med. 2009;361(23):2209-2220.

6. Robb ML, et al. Risk behaviour and time as covariates for efficacy of the HIV vaccine regimen ALVAC-HIV (vCP1521) and AIDSVAX B/E: a post-hoc analysis of the Thai phase 3 efficacy trial RV 144. Lancet Infect Dis. 2012;12(7):531-537.

7. Corey L, Gilbert PB, Tomaras GD, Haynes BF, Pantaleo G, Fauci AS. Immune correlates of vaccine protection against HIV-1 acquisition. Sci Transl Med. 2015;7(310):310rv7.

8. Perez LG, et al. V1V2-specific complement activating serum IgG as a correlate of reduced HIV-1 infection risk in RV144. PLoS One. 2017;12(7):e0180720.

9. Chung AW, et al. Polyfunctional Fc-effector profiles mediated by IgG subclass selection distinguish RV144 and VAX003 vaccines. Sci Transl Med. 2014;6(228):228ra38.

10. [No authors listed]. Experimental HIV Vaccine Regimen Ineffective in Preventing HIV. National Institutes of Health Web Site. https://www.nih.gov/news-events/news-releases/experimental-hiv-vaccine-regimen-ineffective-preventing-hiv. Published February 3, 2020. Accessed May 18, 2020.

11. Haynes BF, et al. Immune-correlates analysis of an HIV-1 vaccine efficacy trial. N Engl J Med. 2012;366(14):1275-1286.

12. Yates NL, et al. Vaccine-induced Env V1-V2 IgG3 correlates with lower HIV-1 infection risk and declines soon after vaccination. Sci Transl Med. 2014;6(228):228ra39.

13. Rerks-Ngarm S, et al. Randomized, double-blind evaluation of late boost strategies for HIV-uninfected vaccine recipients in the RV144 HIV vaccine efficacy trial. J Infect Dis. 2017;215(8):1255-1263.

14. Easterhoff D, et al. Boosting of HIV envelope CD4 binding site antibodies with long variable heavy third complementarity determining region in the randomized double blind RV305 HIV-1 vaccine trial. PLoS Pathog. 2017;13(2):e1006182.

15. Alter G, Malenfant JM, Altfeld M. CD107a as a functional marker for the identification of natural killer cell activity. J Immunol Methods. 2004;294(1-2):15-22.

16. Aktas E, Kucuksezer UC, Bilgic S, Erten G, Deniz G. Relationship between CD107a expression and cytotoxic activity. Cell Immunol. 2009;254(2):149-154.

17. Lin L, et al. Human NK cells licensed by killer Ig receptor genes have an altered cytokine program that modifies CD4+ $4^{+}$cell function. J Immunol. 2014;193(2):940-949.

18. Forthal DN. Functions of antibodies. Microbiol Spectr. 2014;2(4):1-17.

19. Gray GE, Michael NL. Cautious optimism for HIV vaccine science. Curr Opin HIV AIDS. 2013;8(5):367-368.

20. Damelang T, Rogerson SJ, Kent SJ, Chung AW. Role of IgG3 in infectious diseases. Trends Immunol. 2019;40(3):197-211.

21. Ackerman ME, et al. Polyfunctional HIV-specific antibody responses are associated with spontaneous HIV control. PLoS Pathog. 2016;12(1):e1005315.

22. Phillips B, et al. Adjuvant-dependent enhancement of HIV Env-specific antibody responses in infant rhesus macaques. $J$ Virol. 2018;92(20):e01051-18.

23. Boudreau CM, Yu WH, Suscovich TJ, Talbot HK, Edwards KM, Alter G. Selective induction of antibody effector functional responses using MF59-adjuvanted vaccination. J Clin Invest. 2020;130(2):662-672.

24. Barouch DH, et al. Protective efficacy of adenovirus/protein vaccines against SIV challenges in rhesus monkeys. Science 2015;349(6245):320-324.

25. Barouch DH, et al. Protective efficacy of a global HIV-1 mosaic vaccine against heterologous SHIV challenges in rhesus monkeys. Cell. 2013;155(3):531-539.

26. Liljeroos L, Malito E, Ferlenghi I, Bottomley MJ. Structural and computational biology in the design of immunogenic vaccine antigens. J Immunol Res. 2015;2015:156241 
27. Brown EP, et al. High-throughput, multiplexed IgG subclassing of antigen-specific antibodies from clinical samples. $J$ Immunol Methods. 2012;386(1-2):117-123.

28. Ackerman ME, et al. A robust, high-throughput assay to determine the phagocytic activity of clinical antibody samples. $J$ Immunol Methods. 2011;366(1-2):8-19.

29. Karsten CB, et al. A versatile high-throughput assay to characterize antibody-mediated neutrophil phagocytosis. J Immunol Methods. 2019;471:46-56.

30. Fischinger S, et al. A high-throughput, bead-based, antigen-specific assay to assess the ability of antibodies to induce complement activation. J Immunol Methods. 2019;473:112630.

31. Lu LL, et al. IFN- $\gamma$-independent immune markers of Mycobacterium tuberculosis exposure. Nat Med. 2019;25(6):977-987.

32. Lu LL, et al. A functional role for antibodies in tuberculosis. Cell. 2016;167(2):433-443.e14.

33. Lau KS, Juchheim AM, Cavaliere KR, Philips SR, Lauffenburger DA, Haigis KM. In vivo systems analysis identifies spatial and temporal aspects of the modulation of TNF- $\alpha$-induced apoptosis and proliferation by MAPKs. Sci Signal. 2011;4(165):ra16. 